\title{
Elevated Release of Beta-thromboglobulin and Platelet Factor 4 in Cerebral Infarction Patients with Branch Atheromatous Disease: A Preliminary Report
}

\author{
Akiyoshi YOKOTE, ${ }^{1,2}$ Kazutoshi HASHIMOTO, ${ }^{1}$ Ryu BIKEI, ${ }^{1}$ and Hidetoshi NAKAMOTO ${ }^{1}$ \\ ${ }^{1}$ Department of Neurosurgery, Ebina General Hospital, Ebina, Kanagawa; \\ ${ }^{2}$ Department of Neurosurgery, Tazuke Kofukai Medical Research Institute, \\ Kitano Hospital, Osaka
}

\begin{abstract}
This study evaluated the levels of the platelet activation markers beta-thromboglobulin (beta-TG) and platelet factor 4 (PF4) in patients with branch atheromatous disease (BAD). Patients with newly diagnosed cerebral infarctions were recruited into the study; those with cardiogenic cerebral infarctions were excluded. Beta-TG and PF4 levels were measured before therapeutic intervention and compared between patients with and without BAD; Welch's $t$-test was used to determine significant differences between the groups. A total of 15 subjects were enrolled in the study, and 8 were diagnosed with BAD. Beta-TG $(P=0.031)$ and $P F 4(P=0.041)$ levels were significantly higher in the BAD patients than in the non-BAD patients. Platelet activity is normally elevated in patients with cerebral infarctions, but is elevated to an even greater extent in BAD patients. The evaluation of beta-TG and PF4 levels may be beneficial for the elucidation of BAD.
\end{abstract}

Key words: branch atheromatous disease, beta-thromboglobulin, platelet factor 4, platelet activity

\section{Introduction}

Branch atheromatous disease (BAD), initially reported by Caplan, ${ }^{1)}$ is characterized by the development of atheromatous plaques at penetrating artery branch points in the brain. When these plaques rupture, they may cause peripheral artery occlusions due to dispersion of thrombi into the penetrating branches, complete occlusion of branch orifices, and the spread of lesions to adjacent branch orifices. ${ }^{2)}$

Antiplatelet therapy has been recommended for the treatment of atherothrombotic brain infarctions, lacunar infarctions, and BAD because elevated platelet activity is usually associated with these types of infarctions. The purpose of this study was to confirm the levels of platelet activation markers (beta-thromboglobulin [beta-TG] and platelet factor 4 [PF4]) in patients diagnosed with BAD.

\section{Materials and Methods}

Patients with newly diagnosed cerebral infarctions were included in the study. The included patients

Received February 13, 2015; Accepted April 5, 2015 were ineligible if they had a history of stroke; had been treated with anticoagulants, antiplatelet drugs, or immunosuppressive drugs; had any malignancies, liver diseases, or infections; or if they were using anti-inflammatory drugs. Similarly, patients with atrial fibrillation were excluded from the present study because the recommended therapy for cardiogenic cerebral infarctions is different from that for the above-mentioned types of infarctions.

We measured beta-TG and PF4 levels in patients at the time of their admission, before any treatment, using an immunoassay (SRL Inc., Tokyo). Neurologic activity was measured at the time of admission and 7 days after symptom onset. Furthermore, neurologic changes were characterized using the Barthel index. ${ }^{3)}$

After blood collection, ozagrel sodium (160 mg/day, on days 1-3) and clopidogrel (75 $\mathrm{mg} /$ day) were prescribed to all patients. Some reports recommend the use of argatroban hydrate for the treatment of BAD. ${ }^{4)}$ However, we selected ozagrel sodium for the treatment of our patients, as recommended by the stroke treatment guidelines published by The Japan Stroke Society.

BAD was defined as infarcts in the lenticulostriate arterial (LSA) territory, observed in three or more 
horizontal, cross-sectional magnetic resonance imaging (Philips Achieva 1.5 T instrument, Philips Medical Systems, Best, The Netherlands) slices, and the presentation of characteristic ventral pontine infarcts in the paramedian pontine arterial (PPA) territory, without the presence of major vessel stenosis in the affected side or atrial fibrillation.

Welch's $t$-test was used to calculate the significance of differences between patients diagnosed with BAD and those without BAD; $\mathrm{P}<0.05$ was considered significant.

This observational study was approved by the ethical committee of the hospital where the patients were treated, and the participating individuals provided informed consent.

\section{Results}

A total of 15 newly diagnosed cerebral infarction patients ( 7 men) were enrolled in the study. Based on the definition of $\mathrm{BAD}, 8$ of the subjects $(53 \%$, including 2 men) were diagnosed with BAD. BAD was observed in the LSA territory in 3 patients $(37.5 \%)$ and in the PPA territory in 5 patients $(62.5 \%)$ (Table 1, Fig. 1).
The mean beta-TG levels in the BAD and non-BAD groups were $161.3 \mathrm{ng} / \mathrm{ml}$ (standard deviation [SD], 50.3 ), and $83.6 \mathrm{ng} / \mathrm{ml}$ (SD, 61.9), respectively $(\mathrm{P}=0.031)$. The mean PF4 levels in the BAD and non-BAD groups were $72 \mathrm{ng} / \mathrm{ml}(\mathrm{SD}, 36.6)$ and $31.3 \mathrm{ng} / \mathrm{ml}$ (SD, 31.1), respectively $(\mathrm{P}=0.041)$. Furthermore, the beta-TG level was $>200 \mathrm{ng} / \mathrm{ml}$ in the three patients with BAD in the LSA territory; similarly, PF4 levels were $>100$ in those same patients. These observations suggested a high level of platelet activity. Based on the Barthel indexes, the patients in the BAD group did not demonstrate worsening neurologic symptoms, except patient No. 3 , and demonstrated neurologic symptoms that were of similar severity to those in non-BAD patients.

\section{Discussion}

Increased platelet activity has been previously reported to be associated with cerebrovascular disorders ${ }^{5,6)}$ and elevated PF4 levels were reported in patients with lacunar infarctions in a study comparing patients with lacunar infarctions to healthy individuals. ${ }^{7}$ Furthermore, Ohara et al. reported that patients with large lacunar infarctions

Table 1 Characteristics of 15 patients with cerebral infarctions

\begin{tabular}{|c|c|c|c|c|c|c|c|c|c|c|}
\hline $\begin{array}{l}\text { Patient } \\
\text { No. }\end{array}$ & $\begin{array}{c}\text { Type } \\
\text { of cerebral infarction }\end{array}$ & $\begin{array}{l}\text { Beta TG } \\
(\mathrm{ng} / \mathrm{ml})\end{array}$ & $\begin{array}{c}\mathrm{PF} 4 \\
(\mathrm{ng} / \mathrm{ml})\end{array}$ & $\begin{array}{c}\text { BI } \\
\text { at time of } \\
\text { admission }\end{array}$ & $\begin{array}{c}\text { BI } \\
\text { (after } \\
7 \text { days) }\end{array}$ & HT & HbA1c & DL & Age & Sex \\
\hline 1 & CAE & 45 & 10 & 0 & 0 & $(-)$ & $5.6 \%$ & $(+)$ & 79 & $\mathrm{M}$ \\
\hline 2 & BAD (PPA territory) & 60 & 12 & 45 & 50 & $(-)$ & $4.8 \%$ & $(-)$ & 67 & $\mathrm{~F}$ \\
\hline 3 & BAD (LSA territory) & $>200$ & $>100$ & 70 & 35 & $(+)$ & $5.9 \%$ & $(+)$ & 85 & $\mathrm{~F}$ \\
\hline 4 & BAD (PPA territory) & 137 & 60 & 85 & 85 & $(-)$ & $5.7 \%$ & $(+)$ & 69 & M \\
\hline 5 & CAE & 22 & 7 & 5 & 5 & $(-)$ & $6.9 \%$ & $(+)$ & 91 & M \\
\hline 6 & Lacunar infarction & 53 & 17 & 90 & 95 & $(+)$ & $5.8 \%$ & $(+)$ & 71 & $\mathrm{~F}$ \\
\hline 7 & BAD (PPA territory) & 108 & 26 & 60 & 75 & $(-)$ & $5.8 \%$ & $(-)$ & 85 & M \\
\hline 8 & CAE & $>200$ & $>100$ & 5 & 60 & $(+)$ & $4.9 \%$ & $(+)$ & 82 & $\mathrm{~F}$ \\
\hline 9 & CAE & 61 & 16 & 0 & 0 & $(-)$ & $4.9 \%$ & $(-)$ & 88 & $\mathrm{~F}$ \\
\hline 10 & CAE & 156 & 51 & 90 & 90 & $(+)$ & $5.8 \%$ & $(-)$ & 87 & $\mathrm{~F}$ \\
\hline 11 & BAD (LSA territory) & $>200$ & $>100$ & 55 & 85 & $(-)$ & $5.9 \%$ & $(+)$ & 88 & M \\
\hline 12 & BAD (PPA territory) & 185 & 78 & 100 & 100 & $(+)$ & $5.9 \%$ & $(+)$ & 78 & $\mathrm{~F}$ \\
\hline 13 & BAD (PPA territory) & $>200$ & $>100$ & NA & NA & $(+)$ & $6.2 \%$ & $(+)$ & 63 & $\mathrm{M}$ \\
\hline 14 & BAD (LSA territory) & $>200$ & $>100$ & 80 & 100 & $(+)$ & $5.7 \%$ & $(+)$ & 76 & $\mathrm{~F}$ \\
\hline 15 & Lacunar infarction & 48 & 18 & 60 & 80 & $(+)$ & $6.3 \%$ & $(-)$ & 60 & M \\
\hline
\end{tabular}

BAD: branch atheromatous disease, beta-TG: beta-thromboglobulin, BI: Barthel index, CAE: cerebral atheromatous embolism, DL: dyslipidemia, F: female, HbA1c: glycated hemoglobin, HT: hypertension, LSA: lenticulostriate arterial, M: male, PF4: platelet factor 4, PPA: paramedian pontine arterial. 

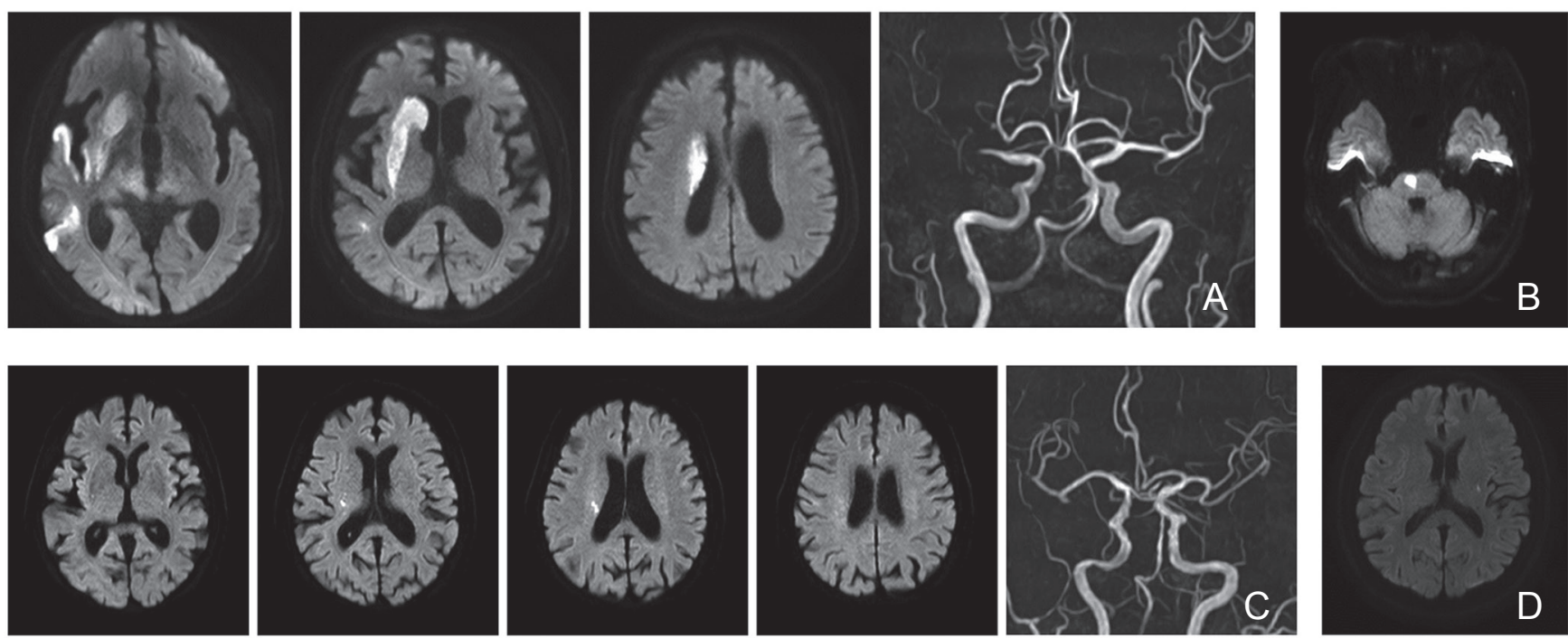

Fig. 1 Representative magnetic resonance imaging scans from patients diagnosed with cerebral infarctions. A: Patient 1 showing a cerebral atheromatous embolism. B: Patient 2 showing cerebral infarction in the paramedian pontine arterial territory (branch atheromatous disease). C: Patient 3 showing cerebral infarction in the lenticulostriate arterial territory (branch atheromatous disease). D: Patient 15 showing a lacunar infarction.

demonstrated beta-TG levels of $46.2 \pm 28.7 \mathrm{ng} / \mathrm{ml}$ and PF4 levels of $11.3 \pm 15.1 \mathrm{ng} / \mathrm{ml} ;{ }^{8)}$ beta-TG values are $<50 \mathrm{ng} / \mathrm{ml}$ and normal $\mathrm{PF}-4$ values are $<20 \mathrm{ng} / \mathrm{ml}$, respectively, in healthy individuals. ${ }^{8}$ Thus, both beta-TG and PF4 levels are known to be elevated in patients with thrombotic disorders, such as peripheral artery disease, coronary artery disease, or cerebral infarction. Furthermore, platelet activity is considered to be an indicator of these thrombotic disorders. Yamamoto et al. reported that treatment involving the use of conventional antithrombotic therapy decreases platelet activity, and leads to an improved treatment effect. ${ }^{4)}$

PF4 possesses some heparin-neutralizing activity, and in a bovine study, beta-TG has been reported to play a role in the inhibition of prostacyclin (PGI2) production. ${ }^{9)}$ The hydrolysis of PGI2 has also been reported to be promoted in patients with cerebral infarctions, and there is a relationship between a decrease in PGI2 production and platelet activity. ${ }^{6,10)}$ This suggests the possibility of a relationship between beta-TG and PF4 values and a tendency for angiostenosis; therefore, these are important findings for the recognition of BAD pathology. The results of the present study suggest that the elevated levels of beta-TG and PF4 in BAD patients further increase the associated platelet activity.

However, beta-TG and PF4 levels are also elevated in patients with diabetes. Additionally, diabetes has been reported to worsen neurologic findings in patients with lacunar infarctions. ${ }^{11)}$ Thus, BAD may tend to develop in patients with elevated beta-TG and PF4 levels. Currently, there is a lack of clarity regarding whether beta-TG and $\mathrm{PF} 4$ increase in $\mathrm{BAD}$ patients or if BAD develops more easily when beta-TG and PF4 levels are elevated. Regardless, platelet activity is elevated in patients diagnosed with BAD. In the present study, we were only able to confirm that platelet activity is significantly higher in BAD patients than in non-BAD patients; we were unable to report a relationship between platelet activity and neurologic deterioration or between platelet activity and BAD pathology. However, exacerbated neurologic symptoms were not evident in the BAD group, relative to the non-BAD group, based on the Barthel index results. The National Institute of Health Stroke Scale (NIHSS) may be a more appropriate measurement tool, for these patients, than the Barthel index. We had originally selected the Barthel index to detect changes in activities of daily living in stroke patients, but were unsuccessful. Thus, we believe that the NIHSS may be more useful than the Barthel index for evaluating fine neurological changes because it includes basic neurological findings, paralysis, and sensory disturbances.

Laboratory determinations of beta-TG and PF4 levels require a week before the results are known. Furthermore, the methods for collecting and preserving blood samples for PF4 and beta-TG level determinations are unique; thus, they are generally not routinely performed. Future assessments of larger numbers 
of patients should be performed using the methods described in this study.

In conclusion, the present study compared platelet activities in patients with cerebral infarctions who were diagnosed with BAD and in those without $\mathrm{BAD}$. Based on the beta-TG and PF4 levels, platelet activity was confirmed to be significantly elevated in the BAD group compared with that in the non-BAD group. Future research to examine and assess changes in beta-TG and PF4 levels and to correlate those changes with clinical manifestations, before and after intervention, may be useful for the elucidation and future treatment of BAD.

\section{Conflicts of Interest Disclosure}

The authors have no financial conflicts of interest to disclose concerning this report and have completed the online self-reported COI Disclosure Statement Forms on the journal's website.

\section{References}

1) Caplan LR: Intracranial branch atheromatous disease: a neglected, understudied, and underused concept. Neurology 39: 1246-1250, 1989

2) Yamamoto Y: [Concept, pathophysiology and treatment for branch atheromatous disease]. Rinsho Shinkeigaku 54: 289-297, 2014 (Japanese)

3) Mahoney FI, Barthel DW: Functional evaluation: the Barthel index. Md State Med J 14: 61-65, 1965

4) Yamamoto Y, Nagakane Y, Makino M, Ohara T, Koizumi T, Makita N, Akiguchi I: Aggressive antiplatelet treatment for acute branch atheromatous disease type infarcts: a 12-year prospective study. Int J Stroke 9: E8, 2014
5) Uchiyama S, Takeuchi M, Osawa M, Kobayashi I, Maruyama S, Aosaki M, Hirosawa K: Platelet function tests in thrombotic cerebrovascular disorders. Stroke 14: 511-517, 1983

6) Akopov S, Darbinian V, Grigorian G, Kosyan A, Gabrielian E: Elevated velocity of prostacyclin degradation in blood as a possible risk factor in patients with cerebrovascular disorders. Eur Neurol 33: 252-255, 1993

7) Ilhan D, Ozbabalik D, Gulcan E, Ozdemir O, Gulbacs Z: Evaluation of platelet activation, coagulation, and fibrinolytic activation in patients with symptomatic lacunar stroke. Neurologist 16: 188-191, 2010

8) Ohara T, Yamamoto Y, Oiwa K, Hayashi M, Nakagawa M: [Clinical classification for lacunar infarct. An investigation of 130 consecutive cases of lacunar infarctions]. Rinsho Shinkeigaku 45: 6-12, 2005 (Japanese)

9) Hope W, Martin TJ, Chesterman CN, Morgan FJ: Human beta-thromboglobulin inhibits PGI2 production and binds to a specific site in bovine aortic endothelial cells. Nature 282: 210-212, 1979

10) Akopov SE, Gabrielian ES: The relationship between the degradation of prostacyclin in blood and antiplatelet drug effects in patients with atherosclerosis. J Clin Pharmacol 33: 1048-1051, 1993

11) Nakamura K, Saku Y, Ibayashi S, Fujishima M: Progressive motor deficits in lacunar infarction. Neurology 52: 29-33, 1999

Address reprint requests to: Akiyoshi Yokote, Department of Neurosurgery, Tazuke Kofukai Medical Research Institute, Kitano Hospital, 2-4-20 Ohgimachi Kita-ku, Osaka 530-8480, Japan. e-mail: Yokote.Akiyoshi@gmail.com 